\title{
Unconventional baryon and meson spectroscopy at Jefferson Lab
}

\author{
M.Battaglieri \\ for the CLAS collaboration
}

Istituto Nazionale di Fisica Nucleare - Via Dodecaneso 3316139 Genova ITALY

\begin{abstract}
Understanding quark and gluon confinement in Quantum Chromodynamics is one of the main issue in hadronic physics. Electromagnetic probes, real and virtual photons, are a powerful tool to investigate how the QCD-partons manifest themselves in strong interaction at the energy scale of the nucleon mass $(\mathrm{GeV})$. The CLAS Collaboration in Hall B at Jefferson Lab (USA) has a broad scientific program ranging from the static and dynamic properties of constituent quarks to the investigation of possible exotic baryon configurations. After a brief description of the CLAS detector, some selected results will be outlined showing the richness of this non-perturbative physics.
\end{abstract}

Keywords: Meson and baryon spectroscopy.

PACS: $13.60 \mathrm{Rj} ; 25.20 \mathrm{Lj}$

\section{INTRODUCTION}

The use of electromagnetic probes in hadron physics has a long history and led to important discoveries: the proton electric and magnetic form factors, the scaling law of deep inelastic scattering etc. Many other experiments were performed thereafter, aiming at the study of particular exclusive channels like pion photo- and electro-production, but mostly with limited statistics and accuracy, due to the low luminosity and duty factors of the machines previously available, as well as to the small detector solid angles. The availability of continuous and high intensity electron and photon beam together with a new large acceptance detector provides coverage over a wide kinematic range for many different final states, including multi-hadron channels.

The CLAS collaboration in Hall B at Jefferson Laboratory has a broad scientific program based on the use of both the primary $\mathrm{CW}$ electron beam and a tagged photon beam. In our kinematics (center of mass energy $W \sim 1-3 \mathrm{GeV}$, photon virtuality $Q^{2} \sim$ $0-5 \mathrm{GeV}^{2}$, and momentum transfer in exclusive reactions $-t \sim 0-6 \mathrm{GeV}^{2}$ ) it is possible to study the transition between the so called 'Soft Physics', where models based on Constituent Quarks or phenomenology are widely applied, to the 'Hard Physics' regime, where perturbative QCD or dimensional analysis are used to interpret the data. In this contribution I will show how the QCD-partons manifest themselves in the baryon spectrum, in vector meson photoproduction and in some quark configurations beyond the standard quark model (pentaquarks). 


\section{CLAS AT JEFFERSON LAB}

The Continuous Electron Beam Accelerator Facility (CEBAF) at Jefferson Lab delivers a low emittance, high resolution, $100 \%$ duty-cycle electron beam to three different experimental halls $\mathrm{A}, \mathrm{B}$ and $\mathrm{C}$, simultaneously. The maximum energy is $5.8 \mathrm{GeV}$ (with $80 \%$ polarization available) with a maximum current of $180 \mu \mathrm{A}$. The Hall B is mainly devoted to experiments that require the detection of several particles in the final state. It hosts the CLAS detector [1] (CEBAF Large Acceptance Spectrometer), built around six superconducting coils producing a toroidal magnetic field. The detector package consists of three layers of drift chambers for track reconstruction, one layer of scintillators for time-of-flight measurements and hadron identification, forward Cherenkov counters for electron-pion discrimination, and electromagnetic calorimeters to identify electrons and neutral particles.

\section{STATIC PROPERTY OF CONSTITUENT QUARKS: THE $N^{*}$ PROGRAM}

One of the features of real and virtual photon interactions with the proton is the appearance of bumps in the inelastic part of the cross section, corresponding to values of the invariant $\mathrm{CM}$ energy $W$ between 1.1 and about $2 \mathrm{GeV}$. This is interpreted as manifestation of excited states of the nucleon (with definite angular momentum and parity), also observed in pion absorption and nucleon-nucleon scattering. Despite progress being made within the framework of quark models to explain the resonances in terms of three quarks, the number of states observed in the spectrum is higher than what predicted by any symmetric quark model based on SU(6). A possible explanation concerns the underlying symmetry: e.g. the Quark Cluster Model, where two of the three constituents stay together in a di-quark configuration leading to a fewer number of predicted states. Another possibility is related to the QCD mixing effects that could decouple many of these states from the pion-nucleon channel, with a consequent lack of evidence in elastic $\pi N$ scattering, while strongly coupling them to multipion channels $(N \pi \pi)$ or higher masses mesons $(N \eta, N \omega, k Y)$. Search for the states still missing in the experimental observation is therefore crucial in understanding the basic degrees of freedom in baryon structure. As an example, I briefly report the CLAS results for the exclusive reaction $e p \rightarrow e^{\prime} p \pi^{+} \pi^{-}$[2]. The new CLAS data show for the first time a clear evidence of the presence of bumps in the total cross section at different $Q^{2}$ values, and a 'missing' strength around $W \sim 1.7 \mathrm{GeV}$ (see fig. 1). The comparison to a phenomenological model incorporating the available knowledge on $N^{*}$ and $\Delta$ states shows an evident discrepancy. A better description of the data is obtained either by a sizable change of the $P_{13}(1720)$ resonance or by introducing a new baryon state not reported in published analysis. 

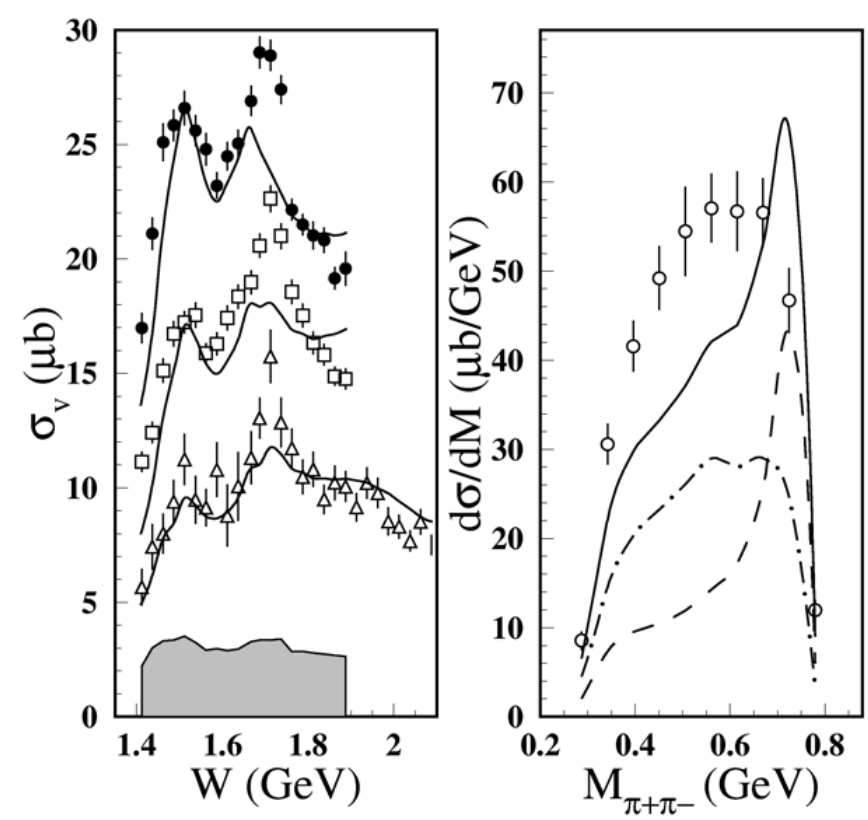

FIGURE 1. Left: Total cross section for $\gamma^{*} p \rightarrow p \pi^{+} \pi^{-}$as a function of $\mathrm{W}$ at $<Q^{2}>\sim 0.65 \mathrm{GeV}^{2}$ (full points), $<Q^{2}>\sim 0.95 \mathrm{GeV}^{2}$ (open squares) and $<Q^{2}>\sim 1.3 \mathrm{GeV}^{2}$ (open triangles). Right: $d \sigma / d M_{\pi^{+} \pi^{-}}$ at $\left\langle Q^{2}>\sim 0.95 \mathrm{GeV}^{2}\right.$. The curves represent a phenomenological model. Dashed line includes all resonances, dot-dashed non resonant part while solid lines are the full calculation.

\section{DYNAMIC PROPERTIES OF CONSTITUENT PARTONS: VECTOR MESON PHOTOPRODUCTION}

One of the key issues in hadronic physics is the study of the interplay between the hadronic (mesons and baryons) and partonic (quarks and gluons) degrees of freedom. Exclusive reactions at large momentum transfer can be used to reduce the hadron complexity in terms of elementary parton scattering. In fact, large momentum transfer (both $Q^{2}$ and $-t$ ) means short distances and, by taking advantage of the fundamental QCD property of the asymptotic freedom, it should be possible to decouple the non perturbative long distance effects from the perturbative short distance interactions. In particular, there are some theoretical arguments supporting the idea that exclusive processes involving one or more photons are, at leading twist, insensitive to long distance physics. Photoreactions at large momentum transfers are ideal tools for unraveling the microscopic nuclear dynamics. At moderate energy and momentum transfer, this reaction can be described using hadronic (meson-baryon) degrees of freedom. As higher energies and momenta are reached this picture fails and the partonic degrees of freedom emerges.

The known forward-peaked cross section, traditionally interpreted in terms of diffractive production, can be parametrized by a hard mechanism at high momentum transfer, where exchange of gluon pairs can take place. Beside the 'QCD-inspired' two-gluon 

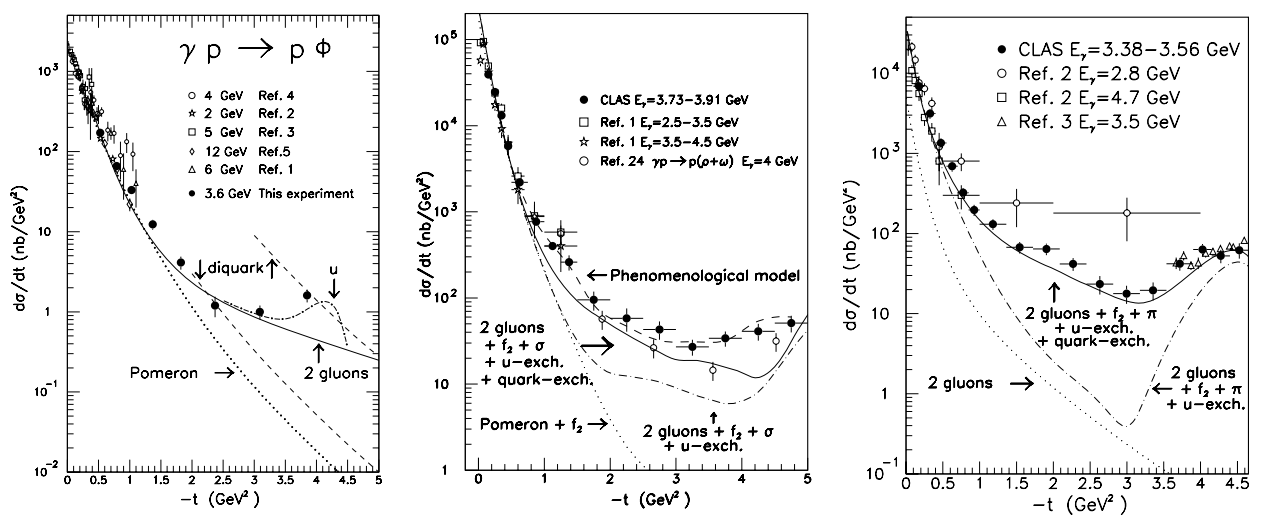

FIGURE 2. Differential cross sections for vector meson photoproduction at CLAS: $\phi$ (left) $\rho$ (middle) and the $\omega$ (right).

exchange, other microscopic mechanisms such as quark exchange or quark correlations can also be investigated. CLAS experiments have measured the $t$ dependence of the photoproduction of $\phi[3], \rho$ [4], and $\omega$ [5] on the proton up to values around $5 \mathrm{GeV}^{2}$ where, previously, only scarce data were available for the $\rho$ and the $\omega$ and no data existed for $\phi$ production at $-t>1 \mathrm{GeV}^{2}$. Figure 2 show the experimental results compared with a QCD-inspired model that successfully reproduces the three reactions. In the $\phi$ channel, due to the dominant $s \bar{s}$ component, the exchange of quarks is strongly suppressed. In fact, for $-t>2 \mathrm{GeV}^{2}$ the data rule out the diffractive Pomeron exchange, and its twogluon realization alone is able to reproduce the data. In the calculation the gluons can couple to any quarks in the baryon, and quark correlations in the proton are taken into account. The two-gluon exchange mechanism alone badly misses the cross section at large $-t$ for the $\rho$ and the $\omega$ photoproduction indicating that other reaction mechanisms are needed. Good agreement with data is obtained when quark-exchange processes are added to the calculation.

\section{BEYOND THE STANDARD QUARK MODEL: THE PENTAQUARK SEARCH}

All known hadronic matter is composed of two kinds of quark configurations: baryons, such as protons and neutrons, which are combinations of 3 quarks (qqq), and mesons, such as pions, which are combinations of a quark and an anti-quark $(q \bar{q})$. However, states of matter consisting of four quarks and an anti-quark $(q q q q \bar{q})$, called pentaquarks, are expected by the theory of Quantum Chromodynamics. In particular, states where the anti-quark has a different flavor with respect to the others have quantum numbers that are not allowed for standard particles and clearly reveal their 'exotic' nature. In the past, experimental searches focused on the search for pentaquark states, did not provide any clear evidence, leading to the conclusion that discoveries in this field would not be 

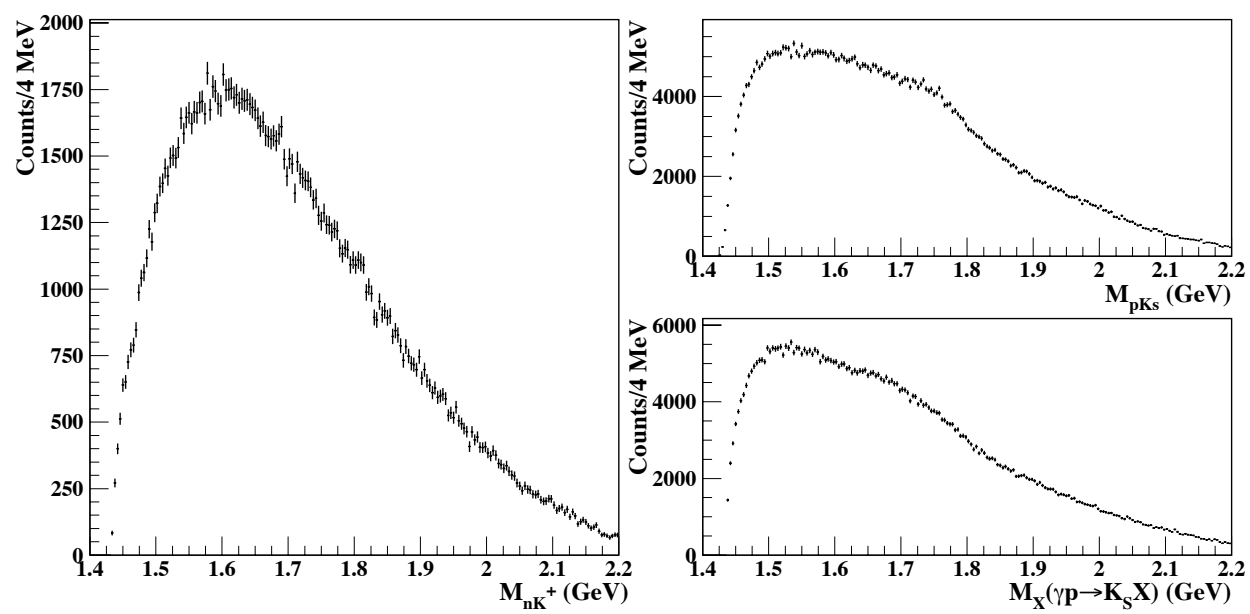

FIGURE 3. Left: the $n K^{+}$invariant mass spectrum for the reaction $\gamma p \rightarrow \bar{K}^{0} K^{+} n$. Right: the $p K_{S}$ invariant mass (top) and $K_{S}$ missing mass (bottom) for the reaction $\gamma p \rightarrow \bar{K}^{0} K^{0} p$. All plots were obtained by integrating over all measured photon energy and angles.

possible in the short term. As a consequence, this particular experimental program was abandoned and no further data analysis was pursued for many years. However, the lack of experimental proof left an open question about the physical manifestation of lowenergy QCD. In spite of that, theoretical interest in this field has continued and, recently, Diakonov and collaborators [6] made definite predictions about the masses and widths of a decuplet of pentaquark states (the so-called "antidecuplet"). The most intriguing aspect of such a multiplet is the presence of three states with exotic quantum numbers: the $\Theta^{+}, S=+1$, and the $\Xi^{--}$and $\Xi^{+}$with $S=-2$. In particular, the $S=1 \Theta^{+}$was unobserved in the baryon sector and is incompatible with a $q q q$ state and requires a pentaquark configuration of the type $u u d d \bar{s}$. The mass of the $\Theta^{+}$was predicted to be quite low $(1540 \mathrm{MeV})$ and its widths very narrow ( $\sim 10-15 \mathrm{MeV})$ implying that if such state exists it should be directly visible in the measured invariant masses without need for more sophisticated Partial Wave Analysis. The first evidence of a $\Theta^{+}$candidate was reported in October 2002 by the LEPS Collaboration, based on the re-analysis of existing data [7]. Several other low-energy experimental groups followed reporting evidence of a peak in the mass range 1521-1555 MeV. While pentaquark signals observed in each experiment suffered from low statistics, the observations in many different reactions using different probes (photons, electrons, protons, neutrinos) and targets (protons, neutrons, nuclei) supported the pentaquark's existence. On the contrary, subsequent re-analysis of data collected in a different set of experiments found no evidence of pentaquarks casting doubts about their existence. A second generation of dedicated experiments $[8,9,10]$, optimized for the pentaquark search, was undertaken at Jefferson Lab. These experiments covered the few GeV region in photon energy, where most of the positive evidence was reported, and collected at least an order of magnitude more data than used in the previous measurements.

In particular, the reactions $\gamma p \rightarrow \bar{K}^{0} K^{+} n$ and $\gamma p \rightarrow \bar{K}^{0} K^{0} p$ were studied in search of 
evidence of the $\Theta^{+}$pentaquark in the $n K^{+}$and $p K^{0}$ decay channels. The final states were isolated by detecting the $K^{+}$or proton, the $K_{S}$ via its decay to $\pi^{+} \pi^{-}$and identifying the neutron or the second neutral kaon with the missing mass technique. For the former decay mode, the direct measurement of the $K^{+}$allows the definition of the strangeness of any resonance observed in this final state. A total of 160,000 and 550,000 events were selected for the reaction $\gamma p \rightarrow \bar{K}^{0} K^{+} n$ and $\gamma p \rightarrow \bar{K}^{0} K^{0} p$ respectively, after the exclusion of background reactions. The $\Theta^{+}$was searched for as a narrow resonance in the $n K^{+}$and $p K_{S}$ mass spectra with a width of 3-4 MeV corresponding to the CLAS resolution for these channels in the kinematic region of this experiment. The two mass spectra are shown in Fig. 3: both of them are smooth and structureless. No evidence for a narrow resonance was found in the mass range 1520-1600 MeV. Combining the results of the two decay modes, we set an upper limit of $0.7 \mathrm{nb}(95 \%$ confidence level) on the total production cross section for the reaction $\gamma p \rightarrow \bar{K}^{0} \Theta^{+}(1540)$. This contradicts the results previously reported for a resonance in the reaction channel $\gamma p \rightarrow \bar{K}^{0} K^{+} n$. The accuracy in the mass determination was found to be $1-2 \mathrm{MeV}$ from the comparison of the measured masses of known particles with world data. The quality of the data and the analysis procedures were tested by deriving the differential and the total cross section for some known reactions and obtaining an agreement within the experimental errors with existing measurements. The same conclusions were found by several independent analyses, giving confidence in our final results. Due to the loose hardware trigger of the experiment, the same data set was analyzed to study the reactions $\gamma p \rightarrow \bar{K}^{*} \Theta^{+}$ and $\gamma p \rightarrow K^{-} \Theta^{++}$[11]. These findings, together with the results coming from other pentaquark search experiments at Jefferson Lab, could clear up the debate about the existence of the pentaquark.

\section{CONCLUSIONS}

Electromagnetic reactions provide insight into the world of hadronic structure, ranging from different aspects in the excitation of nucleon resonances to the partonic picture of hard processes. The large and broad experimental effort of the CLAS collaboration at Jefferson Laboratory is providing a wealth of new data that will help to clarify our understanding of nucleon structure and nuclear dynamics in the intermediate energy region, which is the domain of non-perturbative QCD. The new data put stronger constraints on QCD-inspired calculations showing that this kinematical region can be described as a non perturbative partonic regime, where microscopic degrees of freedom (gluons and quarks) are combined with a low-energy picture of hadrons (constituent quarks, dressed gluons). 


\section{REFERENCES}

1. B. Mecking et al., Nucl. Instrum. and Meth. A503, 513 (2003).

2. M. Ripani et al., Phys. Rev. Lett. 91, 022002 (2003).

3. E. Anciant et al., Phys. Rev. Lett. 85, 4682 (2000).

4. M. Battaglieri et al., Phys. Rev. Lett. 87, 172002 (2001).

5. M. Battaglieri et al., Phys. Rev. Lett. 90, 022002 (2003).

6. D. Diakonov, V. Petrov and M. Polyakov, Z. Phys. A 359, 305 (1997).

7. T. Nakano et al. (LEPS Collaboration), Phys. Rev. Lett. 91, 012002 (2003).

8. M. Battaglieri et al. (CLAS Collaboration), Phys. Rev. Lett.96, 042001 (2006).

9. R. De Vita et al. (CLAS Collaboration), Phys. Rev. D74, 032001 (2006).

10. B. McKinnon et al. (CLAS Collaboration), Phys. Rev. Lett.96, 212001 (2006).

11. V. Kubarovsky et al. (CLAS Collaboration), submitted to Phys. Rev. Lett., arXiv:hep-ex/060500 